\title{
Multi-Criteria and Dynamic Braess's Paradox in Network Systems
}

\author{
Petr FIALA \\ University of Economics, Prague, Czech Republic \\ pfialaevse.cz
}

\begin{abstract}
Networking systems are becoming increasingly important in today's globalization conditions. Network systems show positive and negative effects and paradoxes. One of the paradoxes examined is Braess's paradox, which describes the situation when the addition of edges in the network can lead to a deterioration in the properties of the network system. The paper focuses on generalizing Braess's paradox. One way of generalizing is to consider multiple criteria. An example is analyzed when adding a zero-valued edge according to another criterion worsens the values of both criteria. The second way of exploration is the dynamism of Braess's paradox. Both of these generalizations lead to an even stronger versions of Braess's paradox than by the original problem.
\end{abstract}

Keywords: Networking System, Braess's Paradox, Multiple Criteria, Dynamism.

\section{$1 \quad$ Introduction}

Many of the current network systems are characterized by both the breadth and the complexity of network topology [2]. Overloading plays a growing role not only in transport networks but also in telecommunication networks. Complementarity becomes a decisive factor in information markets. Networks show positive effects. The product unit value increases with the expected number of units sold. Switching costs are also significant when switching to other services or switching to new technology. The decisions of the network users influence not only themselves, but also other participants in the size of profit and cost, timeliness of supply, environmental quality, etc. Paradox situations arise on the networks. Classical paradoxes include Braess's paradox. The paper focuses on generalizing it for multiple criteria and for its dynamic versions.

\section{Braess's Paradox}

Behavior of network participants may be non-cooperative. An example may be the behavior of users of transport or telecommunication networks, where optimization from individual users does not lead to optimization of the whole system. This situation is 
illustrated by the well-known Braess's paradox, when adding another connection by the same demand for service leads to an increase in costs for users [1].

We will analyze a concrete example. The original network in Fig. 1 is composed of four nodes 1, 2, 3, 4 and four edges $h_{1}, h_{2}, h_{3}, h_{4}$, the start and the end of the network consists of nodes 1 and 4 . There are two paths between the start and end of the network $C_{1}=\left\{h_{1}, h_{3}\right\}$ and $C_{2}=\left\{h_{2}, h_{4}\right\}$. Let's assume that the cost of individual edges depends on the size of the flows

$$
n\left(h_{1}\right)=10 x_{1}, n\left(h_{2}\right)=x_{2}+50, n\left(h_{3}\right)=x_{3}+50, n\left(h_{4}\right)=10 x_{4}
$$

and the total required network flow $X=6$.
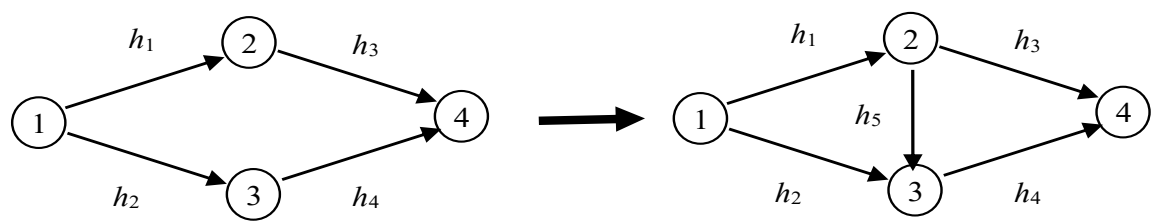

Fig. 1. Braess's paradox.

In the case of optimization from a user's point of view, the equilibrium solution is given by the situation when all the paths between the start and end of the network have the same minimum costs and therefore users are not interested in changing flows on the path. The equilibrium solution is given by the flows on the edges

$$
x_{1}^{*}=3, x_{2}^{*}=3, x_{3}^{*}=3, x_{4}^{*}=3
$$

and by the corresponding costs on the paths

$$
n\left(C_{1}\right)=83, n\left(C_{2}\right)=83 .
$$

After changing the network by adding the edge $h_{5}$ between nodes 2 and 3 with the cost $n\left(h_{5}\right)=x_{5}+10$, the next path $C_{3}=\left\{h_{1}, h_{5}, h_{4}\right\}$ will be created and the equilibrium solution in the original network will no longer be equilibrium in this situation. The equilibrium is defined by the same cost for all paths. In our example, the equilibrium solution will send a flow size of 2 units on each of the three paths, and hence the flows on individual edges

$$
x_{1}^{*}=4, x_{2}^{*}=2, x_{3}^{*}=2, x^{*}{ }_{4}=4, x_{5}^{*}=2
$$

and the corresponding costs on the paths

$$
n\left(C_{1}\right)=92, n\left(C_{2}\right)=92, n\left(C_{3}\right)=92 .
$$


The cost has increased for each network user from 83 to 92 . This increase is also due to the fact that the edges $h_{1}$ and $h_{4}$ are shared by two paths and increase the flow and cost. Braess's paradox is triggered by the network topology as well as the behavior of the participants, which is aimed at optimizing from an individual user perspective. If a path is added between the start and the end of the network that does not share the edges with the original link, then Braess's paradox does not occur.

\section{Multi-Criteria Braess's Paradox}

Multi-criteria formulation can also be considered. The role of costs can be extended, for example, to the consideration of emissions associated with edge flows. Adding a zero-emission edge leads to an increase in total emissions without changing the total flow [3].

Consider an example from the original network in Fig. 1, with the same assignment. Suppose, moreover, that the emissions at individual edges are dependent on the size of the flows

$$
e\left(h_{1}\right)=2 x_{1}, e\left(h_{2}\right)=x_{2}, e\left(h_{3}\right)=x_{3}, e\left(h_{4}\right)=2 x_{4} .
$$

For the total required network flow $X=6$, the original equilibrium solution is given by the flows at the edges

$$
x_{1}^{*}=3, x_{2}^{*}=3, x_{3}^{*}=3, x_{4}^{*}=3
$$

and total emissions are

$$
E=2(3)+1(3)+1(3)+2(3)=18 .
$$

After changing the network by adding the edge $h_{5}$ between nodes 2 and 3 with zero emissions, the equilibrium solution will be given by edge flows

$$
x_{1}^{*}=4, x_{2}^{*}=2, x_{3}^{*}=2, x_{4}^{*}=4, x_{5}^{*}=2
$$

and total emissions will increase

$$
E=2(4)+1(2)+1(2)+2(4)=20 .
$$

Adding a new zero-emission edge will not only increase costs for all participants but also the amount of emissions generated.

Our example demonstrated that adding a zero-valued edge for another criterion may worsen values for both criteria. General consideration of multiple criteria and the search for equilibrium solutions with multiple criteria will be the subject of further research. 


\section{Dynamic Braess's Paradox}

It is possible to consider the dynamic version of Braess's paradox [4]. We will proceed from the situation described in Fig. 1 after the change that occurred by adding the edge $h_{5}$. Now, however, the total flow $X(t)$ will depend on time. In our example,

$$
X(t)=t, t \in\langle 0, T\rangle
$$

and there will be time-dependent flows at each edge

$$
x_{1}(t), x_{2}(t), x_{3}(t), x_{4}(t), x_{5}(t)
$$

with costs that are function of time-dependent flows

$$
\begin{gathered}
n\left(h_{1}\right)=10 x_{1}(t), n\left(h_{2}\right)=x_{2}(t)+50, n\left(h_{3}\right)=x_{3}(t)+50, \\
n\left(h_{4}\right)=10 x_{4}(t), n\left(h_{5}\right)=x_{5}(t)+10, t \in\langle 0, T\rangle .
\end{gathered}
$$

Due to network topology and linear cost functions, this dynamic balance case is easy to solve. General cases can be solved using evolutionary variation inequalities.

There are 3 paths in the network $C_{1}=\left\{h_{1}, h_{3}\right\}, C_{2}=\left\{h_{2}, h_{4}\right\}$ and $C_{3}=\left\{h_{1}, h_{5}, h_{4}\right\}, 3$ equilibrium solutions for the total flow $t$ :

1. Only the path $C_{3}$ with the flow size $t$. Flows at each edge

$$
x_{1}(t)=t, \quad x_{2}(t)=0, \quad x_{3}(t)=0, \quad x_{4}(t)=t, \quad x_{5}(t)=t
$$

with the cost of this path

$$
n\left(C_{3}\right)=21 t+10 .
$$

2. Paths $C_{1}$ and $C_{2}$ with a flow of $\frac{t}{2}$ on each path. There are flows at each edge

$$
x_{1}(t)=\frac{t}{2}, x_{2}(t)=\frac{t}{2}, x_{3}(t)=\frac{t}{2}, x_{4}(t)=\frac{t}{2}, x_{5}(t)=0
$$

with the cost of each path

$$
n\left(C_{1}\right)=n\left(C_{2}\right)=\frac{11}{2} t+50 .
$$

3. Paths $C_{1}$ and $C_{2}$ with the flow of size $\frac{11}{13} t-\frac{40}{13}$ on each path and $C_{3}$ path with the flow $\frac{80}{13}-\frac{9}{13} t$. Flows on individual edges

$$
\begin{gathered}
x_{1}(t)=\frac{11}{13} t+\frac{40}{13}, \quad x_{2}(t)=\frac{11}{13} t-\frac{40}{13}, \quad x_{3}(t)=\frac{11}{13} t-\frac{40}{13}, \\
x_{4}(t)=\frac{2}{13} t+\frac{40}{13}, \quad x_{5}(t)=\frac{80}{13}-\frac{9}{13} t,
\end{gathered}
$$

with the cost of each path 


$$
n\left(C_{1}\right)=n\left(C_{2}\right)=n\left(C_{3}\right)=\frac{31}{13} t+\frac{1010}{13}
$$

By analyzing the dynamically expressed costs it is possible to divide the interval for the total flow $t$ into three intervals:

1. For $t \in\left[0, \frac{80}{31}\right]$ only path $C_{3}$ is used, which uses the added new edge $h_{5}$.

2. For $t \in\left(\frac{80}{31}, \frac{80}{9}\right]$ all three paths are used, including the new path $C_{3}$, and Braess's paradox is emerging.

3. For $t \in\left(\frac{80}{9}, \infty\right)$ only old paths $C_{1}$ and $C_{2}$ are used and the added edge $h_{5}$ is not used at all.

The dynamic version of the problem has shown an even stronger version of Braess's paradox, for some values of the dynamic total flow Braess's paradox is emerging and for some values of the dynamic total flow even a new path will never be used.

\section{Conclusions}

Many of the current economic systems have network connections. These network systems show various interesting effects and paradoxes. Braess's paradox shows an interesting situation that arises by adding another connection in the network system leads to an increase in costs for users by the same demand for service. The paper analyzes the multi-criteria and dynamic versions of Braess's paradox. Analyzed generalizations show even stronger versions of Braess's paradox. The results of the analysis have implications for the practical solution of problems in network systems. These generalizations will be a topic for further research.

Acknowledgements. This paper was supported by the Czech Science Foundation project No. P402/12/G097 DYME - Dynamic Models in Economics and by the grant No. IGA F4/57/2017, Faculty of Informatics and Statistics, University of Economics, Prague.

\section{References}

1. Braess, D.: Über ein Paradoxon aus der Verkehrsplanung. Unternehmensforschung 12(2), 258-268 (1968). DOI: 10.1007/BF01918335.

2. Fiala, P.: Dynamické vytváření cen a alokace zdrojů v sítích. Professional Publishing, Praha (2016).

3. Nagurney, A., Dong, J.: Supernetworks: Decision-Making for the Information Age. 1st edn. Edward Elgar Publishers, Cheltenham (2002).

4. Nagurney, A.: Supply Chain Network Economics: Dynamics of Prices, Flows and Profits. 1st edn. Edward Elgar Publishers, Cheltenham (2006). 